\title{
Inter-generational Consequences for Growing C.elegans in Liquid
}

Itamar Lev ${ }^{\#, 1}$, Roberta Bril ${ }^{1}$, Yunan Liu ${ }^{1}$, Lucila Inés Ceré ${ }^{1}$, and Oded Rechavi, ${ }^{\#, 1,2}$

${ }^{1}$ Department of Neurobiology, Wise Faculty of Life Sciences \& Sagol School of

Neuroscience, Tel Aviv University, Tel Aviv, Israel 69978.

${ }^{2}$ Center for Regenerative and Developmental Biology and Department of Biology, Tufts

University, 200 Boston Avenue, Suite 4600, Medford, MA 02155, USA

\#Corresponding authors: itamai.et@gmail.com and odedrechavi@gmail.com

\section{Abstract:}

In recent years, studies in Caenorhabditis elegans nematodes have shown that different stresses can generate multigenerational changes. Here we show that worms that grow in liquid media, and also their plate-grown progeny, are different from worms whose ancestors were grown on plates. It has been suggested that C.elegans might encounter liquid environments in nature, although actual observations in the wild are few and far between. In contrast, in the lab, growing worms in liquid is commonplace, and often used as an alternative to growing worms on agar plates, to control the composition of the worms' diet, to starve (and synchronize) worms, or to grow large populations for biochemical assays. We found that plate-grown descendants of M9 liquid media-grown worms were longer than control worms, and the heritable effects were apparent already very early in development. We tested for the involvement of different known epigenetic inheritance mechanisms, but could not find a single mutant in which these intergenerational effects are canceled. While we found that growing in liquid always leads to inter-generational changes in the worms' size, trans-generational effects were found to 
be variable, and in some cases the effects were gone after 1-2 generations. These results demonstrate that standard cultivation conditions in early life can dramatically change the worms' physiology in adulthood, and can also affect the next generations.

\section{Introduction}

"You could not step twice into the same river" (Heraclitus)

In comparison to Mendelian inheritance, which entails faithful transmission of discreet packets of information which do not blend or dilute [1], heritable epigenetic data is "soft", or "fluid", as it changes as a function of time, and in response to physiological processes [2].

Generally, mechanisms which allow trans-generational epigenetic effects, namely transmission of information to progeny not exposed to the original trigger, are still poorly understood [3]. However, in C.elegans substantial understanding has been gained regarding the molecular mechanisms that allow transmission of responses via small RNAs across multiple generations (Reviewed in: [4]). Further, many studies with C.elegans have demonstrated that different environmental challenges leave a trace in the progeny, for example viral infections [5-7], starvation [8-11], high temperatures [12-14], high osmolality [15,16], and exposure to toxins [15]. Such stressors can lead to short or long-term gene expression alternations and physiological changes. The inheritance of some of these responses is associated with changes in heritable small RNAs $[8,13,14]$ and/or is correlated with alternation in histone modifications (for example, of H3K4me $[15,17]$. 
In the lab, $C$. elegans are commonly grown on agar plates, but when large numbers of worms are needed, worms are often grown in liquid media [18]. C.elegans are grown in liquid for other purposes as well, for example to synchronize the worms' growth at the L1 stage [18], to control for the composition of the worms' diet [19], and sometimes for studying starvation [18]. Liquid media was also used to study how swim exercise affects the worm's physiology [20]. In addition to $C$. elegans, other nematodes, such as the entomopathogenic species Steinernema $[21,22]$ and Heterorhabditis $[23,24]$ are routinely grown in liquid cultures, and recently, growth in liquid medium of different Pristionchus species was shown to generate striking phenotypic differences [25] (see also discussion).

In nature, C. elegans worms are most often isolated from solid decomposing plant material. However, there are rare reports of isolation of $C$. elegans from freshwater as well [26]. In addition, it has been hypothesized, but not yet documented, that $C$. elegans could encounter liquid environments in the wild, in cases of excessive decomposition, especially of fruits [27].

Here, we report that growth of worms in liquid media changes the morphology of the liquid-grown worms and also the morphology of their plate-grown progeny. We found that plate-grown descendants of liquid-grown worms are on average longer than control worms. This morphological change is apparent early during the worms' life, and persists even in spite of experimental synchronization of the worms' development. In addition, the inheritance of this phenotype does not appear to depend on pheromone production or any single known epigenetic inheritance pathway, as we could not find a single mutant in which these inter-generational effects would be canceled. While the effect is robustly inherited to the immediate $\mathrm{F} 1$ progeny, the inheritance to the $\mathrm{F} 2$ and the $\mathrm{F} 3$ generations 
exhibits variability. These results demonstrate that commonplace cultivation conditions can dramatically change the worms' physiology in adulthood, and can also affect the next generations. We suggest that the growth and handling conditions of at least a few generations should be monitored before experimentation begins.

\section{Results}

Even in the lab, C. elegans often encounters different environments, for example, worms are frequently starved or grown in slightly stressful temperatures, either accidentally or intentionally. It is very common to synchronize or control the speed of the worms' growth, by starving them or by switching the growth temperature. Similarly, in many cases, researchers are forced to grow temperature-sensitive worms in low temperatures, or to cultivate the worms in high temperatures to avoid transgene silencing [28]. Contaminations with different pathogens are very common in C.elegans cultures, and while this has not been studied in detail, such infections could also leave heritable imprints [29]. As described above, worms are often grown in liquid in the lab (the standard liquid used is M9 buffer,[18]). In the course of conducting many experiments with liquidgrown worms, we noticed that after growth in liquid medium, the worms exhibit a different morphology, in comparison to plate-grown worms. We first set out to characterize this phenomenon, and to examine whether the effects could be inherited (Figure 1A).

\section{Progeny of liquid-grown worms are longer}

We noticed that worms that grow in liquid become narrow, and that their body length becomes more variable in comparison to plate-grown worms. These observations 
are in accordance with a previous observation [18], and a recent study showing that worms grown in axenic (chemically defined and bacteria-free) liquid medium (specifically the "Habitation and Reproduction" medium used by NASA when growing worms in space [19]) are also narrower and longer than plate-grown worms [30].

We found that the worms' concentration in liquid affects their size $(p<0.0001$, OneWay ANOVA), and that while in low concentrations the liquid-grown worms are longer (Figure 1B and C), in very high concentrations, liquid-grown worms become shorter (perhaps in this condition not enough food is available). As starvation is known to generate heritable effects in C.elegans [8-11], in all the subsequent experiments we grew worms in liquid in low concentrations (maximum 0.05 worms per microliter, see methods). In this concentration, liquid-grown worms are $\sim 22 \%$ longer than plate-grown worms ( $p<0.0001$, Sidak's multiple comparisons test, Figure 1B and C).

Next, we tested whether plate-grown progeny of liquid-grown worms differ in their body length (Figure 1A and B). Interestingly, we found that the F1 progeny of liquidgrown worms were on average $~ 8 \%$ longer than control worms $(p<0.0001$, Two-Way ANOVA, Figure 1B and D). The heritable effect was very robust, repeated across many biological repeats, and was independent of the concentration of the parent worms in the liquid medium (Figure S1, in addition, experiments were replicated by multiple different experimenters).

When placed in liquid media worms change their movement, to a swim-like behavior, which involves continuous "thrashing". Previously this swim-like behavior was used to study the effects of exercise on the worm's physiology [20]. We wondered whether this energetically demanding behavior could be involved in the morphological change 
found in the liquid-grown worms and their plate-grown progeny. To test this we examined the morphology of liquid-grown immobile unc-119 mutants across generations. We found that liquid-grown unc-119 mutants do not become longer in liquid $(p<0.0001$, Two-Way ANOVA, Figure S2A and B). While experimentation with such Unc mutants is challenging (the mutants exhibit higher length variability, and do not grow well in liquid), the plate-grown progeny of liquid-grown mutants were nevertheless longer than controls $(p<0.0001$, Two-Way ANOVA, Figure S2C). These results suggest that swimming in liquid is not required for the intergenerational effect.

Recently, Celen et al analyzed the transcriptome of worms that grew in a special axenic liquid medium [31]. They found that the genes that were up-regulated in axenic media were enriched with genes related to cuticle formation and morphology. Further, some transcriptional changes were also inherited to plate-grown F1 progeny. The authors did not examine whether the morphology of the progeny of the worms that grew in axenic medium changed, however it is possible that these transcriptional changes correspond to the inherited morphological changes that we observe in the progeny of M9 liquid grown worms. 
A

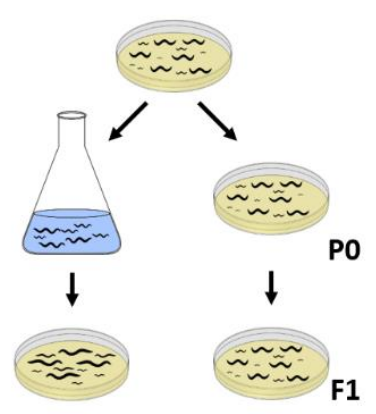

C

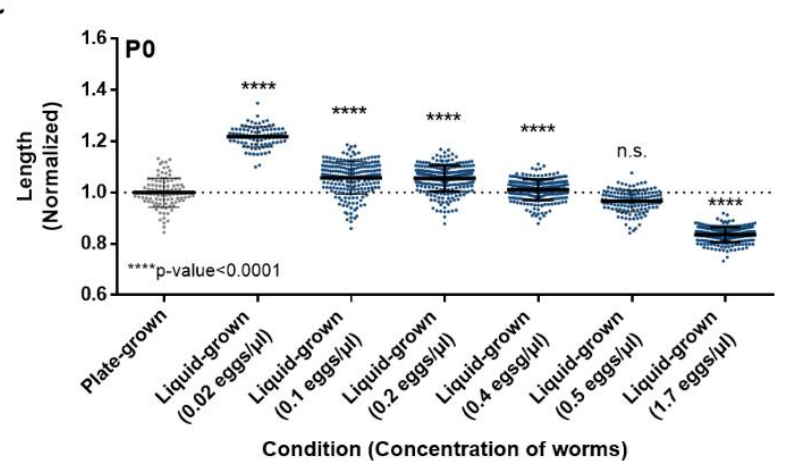

B

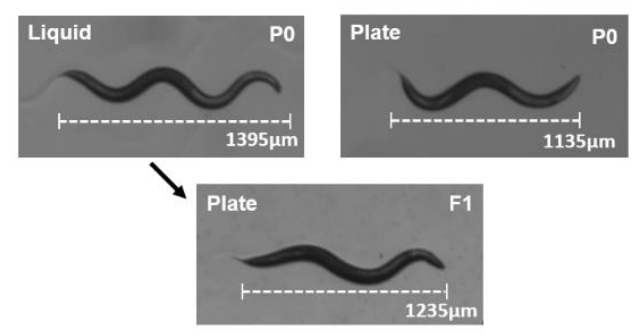

D

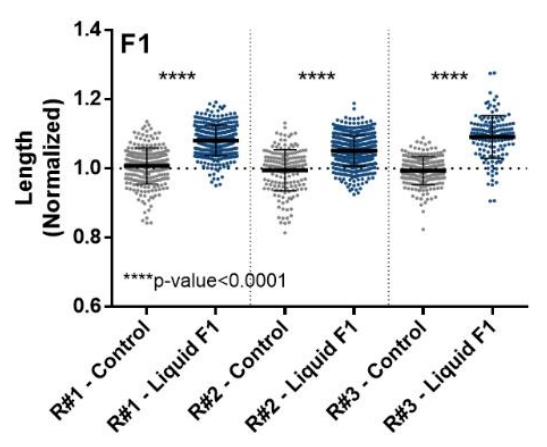

Figure 1. Progeny of liquid-grown worms have longer morphology

(A) Experimental scheme of inter-generational inheritance of growth in liquid medium. Eggs were produced from Isogenic worm lines and placed either on plates or in liquid medium. When the parental P0 worms reached adulthood, they were placed on plates to lay their eggs. When the resulting F1 progeny reached adulthood, their body length was measured.

(B) Representative images of adult plate-grown (P0), liquid-grown (P0) or plate-grown progeny (F1) of liquid-grown worms. The differences in length between the photographed worms match the average length differences found between the examined populations. A scale bar that shows the total stretched length of the worm is presented.

(C) Liquid-grown worms are longer when grown in low concentration. The normalized length of the parental plate-grown, and liquid-grown worms are shown. Worms were grown in liquid in different concentrations (x-axis). The measured length was normalized 
to the plate-grown worms. ${ }^{* \star * *}$-value $<0.0001$, One-way ANOVA, Sidak's multiple comparisons test.

(D) Plate-grown progeny of worms grown in liquid medium are longer. The length of F1 plate-grown progeny of liquid-grown worms and progeny of plate-grown controls are shown. The measured length was normalized to the corresponding control worms. Data from three independent biological repeats are presented ( $N>150$ worms per group). The p-value was determined using Two-way ANOVA with the two categorical variables: A. biological condition (growth in liquid or on plates) and B. biological repeat (independent experiments conducted on separate groups of animals, on different days). Both factors have a significant effect on the measured worm length ( $p$-value $<0.0001$ ). In addition, an interaction effect was found as well ( $p$-value $<0.0001)$. The biological condition explained far more variance than the biological repeat or the interaction $(32.44 \%$ compared to $2.07 \%$ or $1.69 \%$, respectively).

The change in the morphology of the progeny of liquid-grown worms is apparent early during development, and persists even when the progeny's development is synchronized

It is possible that the progeny of the liquid-grown worms is longer, since these worms develop faster. To test this possibility, we monitored the worms' length throughout development. To our surprise, we found that progeny of liquid-grown worms were longer already as eggs ( $<<0.0001$, Two-Way ANOVA, Figures $2 A)$. The progeny of the liquidgrown worms were also longer immediately after hatching, at the L1 stage (Figures 2B and S3, $p=0.022$, see statistical methods), maintained the elongated morphology 
throughout development, and their rate of development was identical to the control group

(Figures 2B and S3, $p=0.7806$, see statistical methods). Moreover, the effect persisted in adults, even when we synchronized the worms' development at the L1 stage by starvation (for 12 hours, see methods. $\mathrm{p}<0.0001$, Two-way ANOVA, Figure 2C). In summary, the elongated morphology of the F1 progeny of liquid-grown worms does not stem from differences in the rate of development.

A

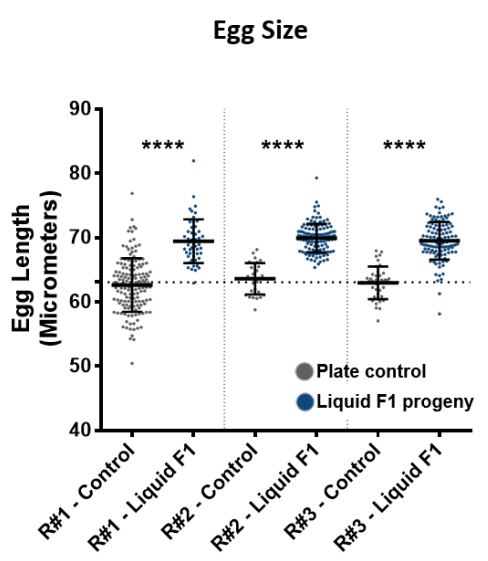

B

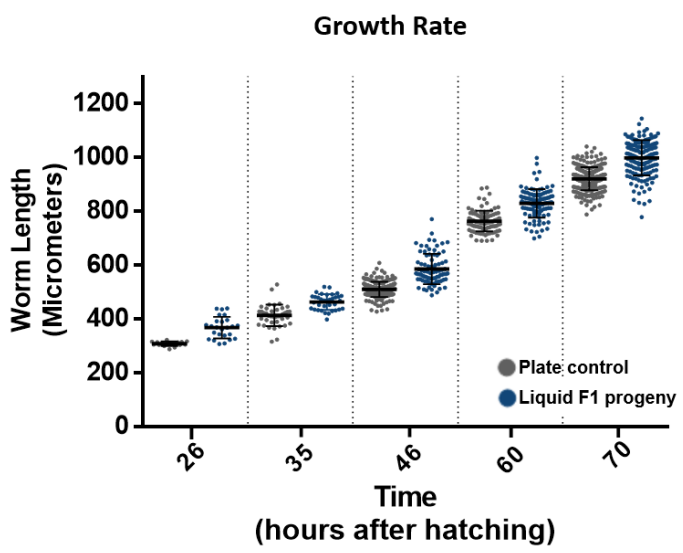

C

Adults synchronized at the L1 staged

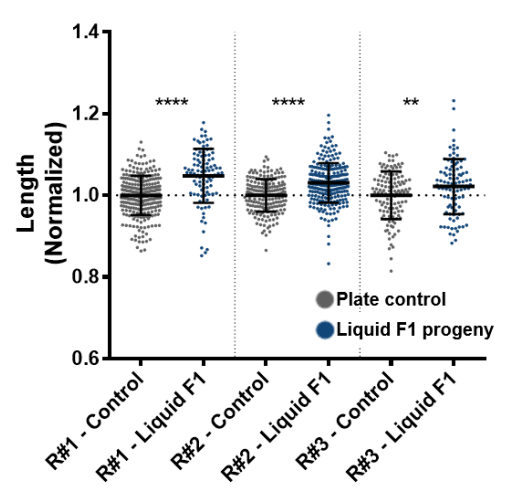

Figure 2. Progeny of liquid-grown worms are longer already early in development.

(A) The length of eggs laid by liquid-grown worms and plate control worms. Data from three independent biological repeats are presented $(N>30) .{ }^{* * *} p$-value $<0.0001$, Two-way ANOVA (biological condition is significant $p<0.0001$, biological repeat and interaction effect factors are not significant: $p=0.8642$ and $p=0.2045$, respectively). Sidak's multiple comparisons test $\mathrm{p}$-value is presented.

(B) The length of progeny of liquid-grown worms and control worms are presented at different stages of the worm's development.

(C) The length of adult worm progeny of liquid-grown and plate-grown controls, 72 hours after being released from starvation-induced developmental arrest at the L1 stage. Data 
from three independent biological repeats are presented $(\mathrm{N}>100) .{ }^{* * *} \mathrm{p}$-value $<0.0001$, ${ }^{* *} p-v a l u e=0.0066$. Two-Way ANOVA (biological condition is significant $p<0.0001$, biological repeat and interaction effect factors are also significant $p=0.0093$. These factors explain $0.81 \%$ of the variance, while the biological condition explain $7.96 \%$ of the variance). Sidak's multiple comparisons test $p$-value is presented. Error bars represent standard deviations.

\section{Progeny of liquid-grown worms are longer even in mutants defective in chemosensation and pheromone biosynthesis.}

In response to various environmental changes, $C$. elegans alter the composition of the molecular social cues (or pheromones) that they secrete [32,33]. In turn, the secreted pheromones greatly affect the worms' physiology [34,35]. For example, in response to crowded culture conditions, C. elegans secrete pheromones which cause starved larval worms to arrest their development and enter the dauer stage (an alternative developmental stage during which worms can endure harsh conditions) [35]. We examined whether sensation of pheromones or other secreted molecules could be the factor that transforms the progeny of the liquid grown worms. To test this, we conducted experiments with mutants defective in pheromone production in general (daf-22) and dauer pheromones sensation specifically (srg-36/37). We also examined mutants that are generally defective in chemosensation (che-2).

DAF-22 is a fatty acid beta oxidase important for the production of short-chain ascarosides [36] and dauer pheromones [37]. SRG-36/37 are two redundant G-proteincoupled receptors for the dauer pheromone ascaroside C3 [38]. Mutations in these genes, 
which confer resistance to dauer formation, were identified in two independent long-term liquid-grown strains (in worms that were grown in liquid for many decades, [38]). We found that liquid-grown daf-22 and srg-36/37 mutants generate a functional intergenerational response and similarly to wild type animals, plate-grown progeny of the liquid-grown mutants were longer than controls (Figure 3). Thus, pheromones are probably not involved in this inter-generational effect.

CHE-2 is a protein with an unknown function that contains G-protein like WD-40 repeats. The sensory neurons' cilia of che-2 mutants are severely defective [39]. Accordingly, these mutants show defects in osmotic pressure avoidance [40], and are defective in chemotaxis to $\mathrm{NaCl}[39,40]$ and to many odorants [41]. We found that progeny of liquid-grown che-2 mutants are also elongated, similarly to wild type worms. Therefore, sensation of secreted molecules does not appear to mediate the intergenerational response that we describe here (Figure 3).

DAF-16 is a transcription factor playing a pivotal role in the insulin/IGF-1 pathway, regulating stress responses, stress survival, longevity, fat metabolism, immunity and dauer formation [42]. DAF-16 was further implicated in inter-generational responses to stress [16]. Surprisingly, plate-grown progeny of liquid-grown daf-16 mutants were elongated, similarly to wild type worms (Figure 3). This suggests that the DAF-16 dependent stress response is not involved in the liquid-induced heritable effect. 


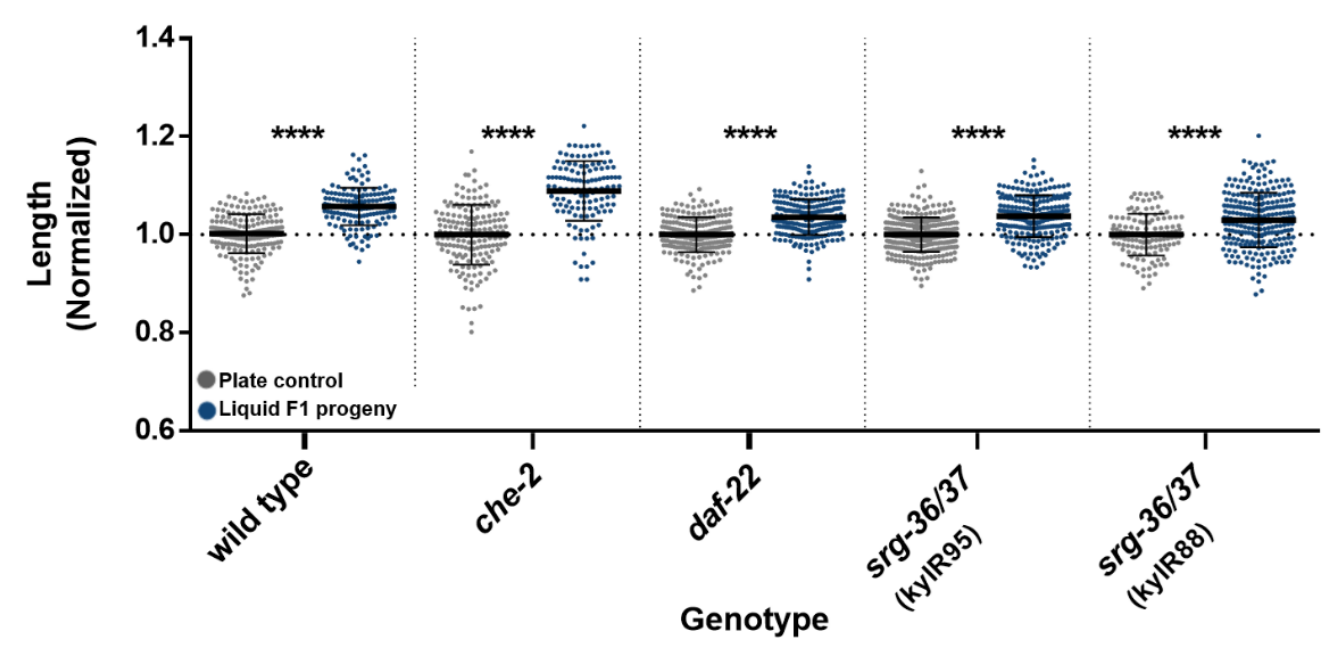

Figure 3. Progeny of liquid-grown worms are longer even in mutants defective in chemosensation and pheromone biosynthesis.

The normalized lengths of progeny of liquid-grown mutants, defective in genes involved in dauer pheromone synthesis (daf-22), pheromone sensation (srg-36/37), stress response (daf-16), and chemosensation (che-2) are presented. $N>100$ per group. ${ }^{* \star * *} p$ value $<0.0001$, One-way ANOVA, Sidak's multiple comparisons test. Error bars represent standard deviations.

Progeny of liquid-grown worms are longer even in mutants defective in inheritance of small RNAs and chromatin modifications

To examine whether the heritable changes in body length depend on inheritance of small RNAs or histone modifications, we examined mutants which are defective in production or inheritance of small RNAs and histone modifications. The nuclear argonautes HRDE-1 and NRDE-3 carry heritable small RNAs, and are required for the inheritance of dsRNA-induced silencing $[43,44]$. While NRDE-3 is expressed in somatic 
tissues and is important for inter-generational inheritance of somatic RNA interference [45], HRDE-1 is expressed in the germline and is required for the trans-generational inheritance (lasting multiple generations) of exogenous, dsRNA-derived small interfering RNAs (siRNAs) and endogenous siRNAs that target germline genes [43]. The amplified siRNAs that are carried by the NRDE-3 and HRDE-1 argonautes are produced by RNA dependent RNA polymerases (RdRP), such as RRF-1. RRF-1 is involved in the inheritance of piRNA-triggered endogenous siRNAs and viral-derived siRNAs $[5,46]$. We found that hrde-1, nrde-3, and rrf-1 mutants, as well as additional mutants defective in siRNA biogenesis (rde-1, rde-4, rrf-3 and mut-16) are not required for the inheritance of the elongated morphology (Figure 4). Previously, Histone-H3-Lysine-9 (H3K9) methylations were implicated in trans-generational inheritance of small RNAs [47-50]. H3K9 mono and di- methylations are deposited by the MET-2 methyltransferase [51], while H3K9me3 is deposited by the SET-25 and SET-32 methyltransferases $[51,52]$. We found that met-2, set-25 and set-32 mutants also inherit the elongated morphology from their liquid-grown parents (Figure 4). To conclude, the inter-generational inheritance of this phenotype does not appear to depend on the canonical epigenetic inheritance pathways that we examined. 


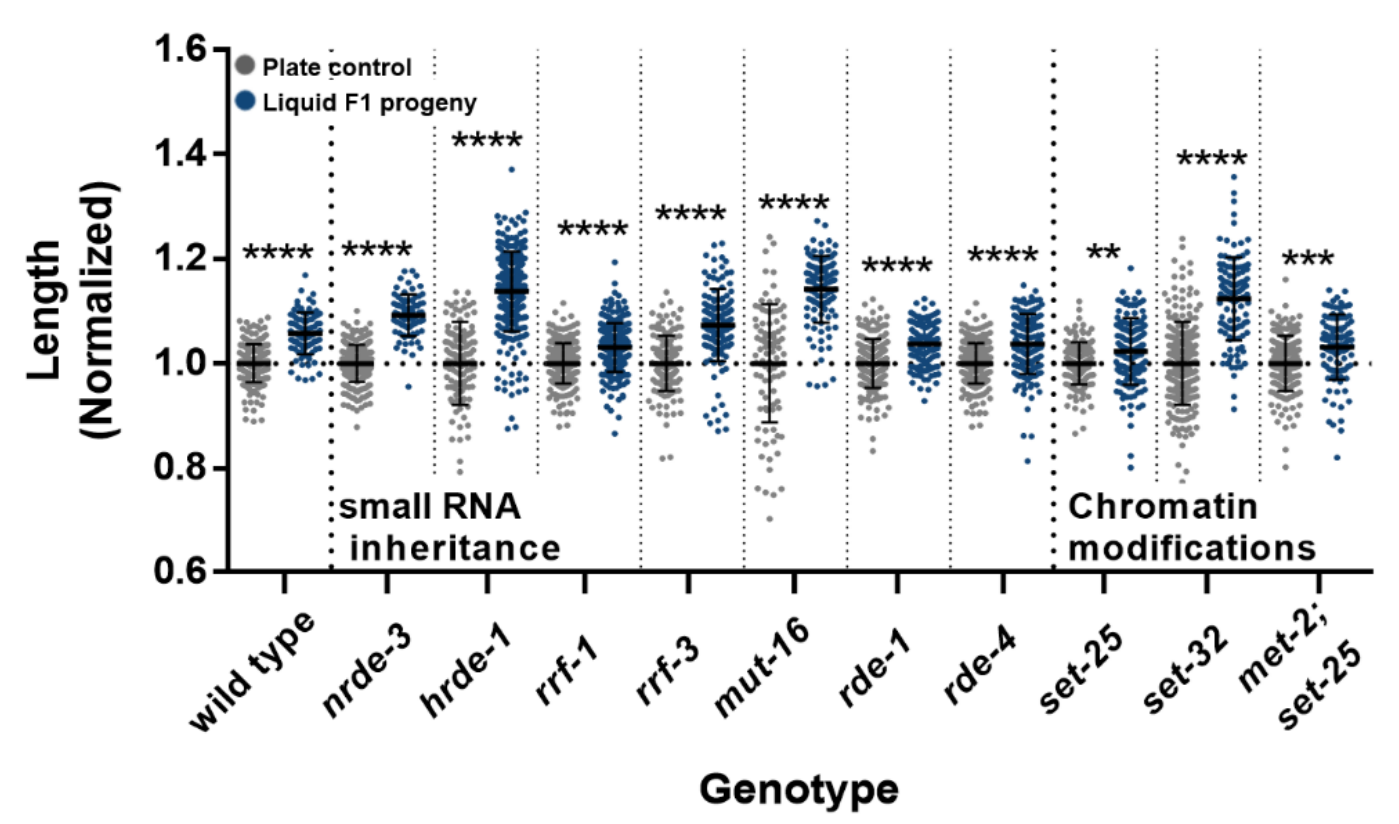

Figure 4. Mutants defective in inheritance of small RNAs and histone methylations are also longer, if they derive from ancestors that were grown in liquid. The normalized lengths of progeny of liquid-grown worms mutant in genes involved in small-RNA biogenesis and inheritance (hrde-1,nrde-3, rrf-1, rrf-3, rde-1, rde-4 and mut16 mutants) or chromatin methylation (set-25,set-32 and met-2;set-25 mutants) are presented. $\mathrm{N}>100$ per group. ${ }^{* * *} \mathrm{p}$-value $<0.0001,{ }^{* * *} \mathrm{p}$-value $=0.0001,{ }^{* *} \mathrm{p}$-value $=0.0084$, One-way ANOVA, Sidak's multiple comparisons test. Error bars represent standard deviations.

Unlike the inter-generational effects, trans-generational inheritance of the morphological changes exhibits variability

Previously, certain environmental stresses, such as L1 starvation [53], starvation during dauer [11], and exposure to high temperatures [12], were shown to induce transgenerational effects that persist for more than two generations. To test whether the 
elongated body morphology can be inherited for more than one generation, we measured the body length of plate-grown worms that derive from liquid-grown parents in the F1, F2 and F3 generations. In contrast to the inter-generational changes to the worms' size that were robustly inherited to the $\mathrm{F} 1$ generation, the trans-generational effect in the $\mathrm{F} 2$ and F3 generations was variable. While in some experiments we did observe transgenerational heritable changes, in other cases the effect was gone after 1-2 generations (Figure 5).

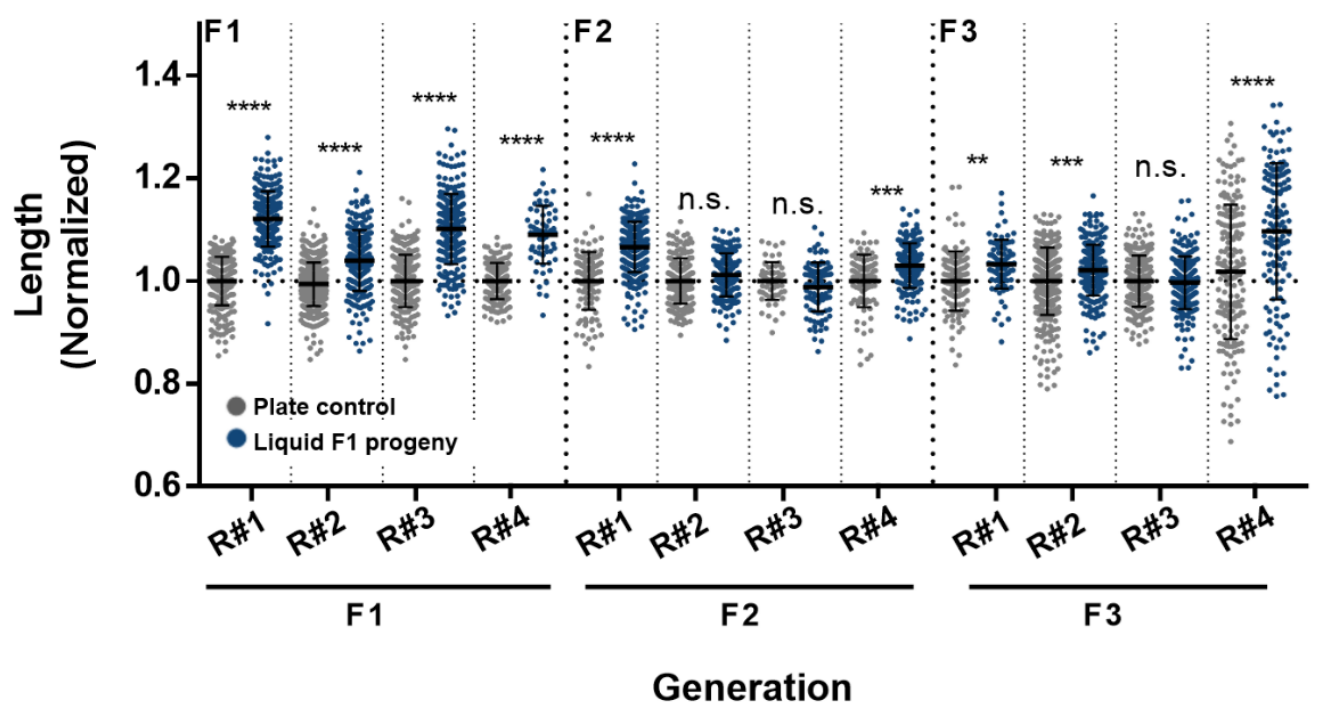

Figure 5. The liquid-induced longer morphology is not always inherited transgenerationally (in some experiments the effects peter out after 1 generation).

The normalized lengths of progeny of liquid-grown worms and progeny of plate-grown controls along the F1, F2 and the F3 generations are presented. The measured length is normalized to the corresponding control worms of the matching generation and biological repeat. Data from four independent biological repeats are presented $(N>150$ worms per group). ${ }^{* * *} p$-value $<0.0001,{ }^{* *} p$-value $<0.005,{ }^{* *} p-v a l u e=0.0015$. One-way ANOVA, Sidak's multiple comparisons test. Error bars represent standard deviations. 


\section{Discussion}

In this manuscript, we show that growth in liquid media under laboratory conditions changes the morphology of the worms and the morphology of their progeny. As briefly described above, there are rare reports of isolation of $C$. elegans from fresh water habitants [26]. There are also phenotypes, which are specific to worms that grow in liquid. For example, infection of $C$. elegans in liquid by the pathogenic bacteria Leucobacter celer was found to induce aggregation of worms (termed "worm stars") [54]. Whether the morphological changes that we describe are adaptive in natural circumstances remains to be determined.

In addition to $C$. elegans other nematodes are routinely cultured in liquid cultures. Entomopathogenic nematodes such as Steinernema [21,22] and Heterorhabditis [23,24] are grown in liquid cultures as means of mass production of a biocontrol agent. However, liquid-grown entomopathogenic nematodes were found to have lower dauer stage recovery rates and pathogenicity $[21,55,56]$, and much effort was invested in optimizing the different aspects of their mass production in liquid cultures [22]. Recently, a different nematode model organism, Pristionchus pacificus, was found to exhibit altered, narrow and elongated morphology when grown in liquid cultures [25]. Interestingly, the dimorphic mouth-form of $P$. pacificus, was found to respond to growth in liquid medium, however, the response was not heritable [25]. Several additional Pristionchus species, but not all, also showed mouth-form alternation in response to growth in liquid culture [25].

During the course of this study we could not identify mutants in which epigenetic pathways are effected, that are defective for the inter-generational inheritance of the elongated morphology. It is possible that such mutants do exist, and could be detected in 
more comprehensive screens. Alternatively, the inter-generational effects that we examined could transmit via many different molecular "agents" that do not necessarily affect epigenetics. Pheromone production and sensation, does not seem to be required for this effect, as mutants in the relevant pathways also exhibited the heritable liquidinduced effect. Changes in other molecules, such as metabolites or hormones, which are not necessarily self-propagating or "amplifiable" (in contrast to heritable small RNAs), could mediate such short term inter-generational inheritance as well.

Previously, environmental changes such as starvation and temperature shifts, both common in the lab, were shown to change the physiology of the worms and of its progeny. Together with growth in liquid, these manipulations should be taken into consideration when designing experiments with $C$. elegans. It is reasonable to assume that many other subtle characters are effected in the progeny of liquid-growth worms (for example behavior). To control for such changes, the worms' growth conditions should be monitored for several generations before experimentation.

Inter-generational and trans-generational inheritance present a unique challenge: to compare between different experiments, the experimenter must keep track of the history of the culture, to make sure different experiments begin from the same starting point. There are currently no standard protocols for achieving this goal. We do not know yet how to "re-set" epigenetic inheritance. Previously, we used CRISPR/cas9 to deactivate HRDE-1, a factor which is important for small RNA inheritance, consequently erasing small RNA memory. This led to the re-setting of the accumulated sterility of chromatin mutants (Mrt phenotype) [49]. Since not all heritable effects are necessarily small RNA-mediated, other re-setting strategies should be devised in parallel. 
The same principles that were discussed above with regard to inter- and transgenerational epigenetic inheritance in $C$. elegans could apply also to other organisms, and even to experiments conducted with cell lines. Several years ago, a controversy arose regarding the mislabeling of many cell lines [57]. While this is certainly a serious problem, it is worth noting that even when the correct cell line is used, cells in culture acquire mutations [58] (and differ in ploidy), and moreover non-genetic inheritance could create variability between cultures in different labs. Indeed, the "generation time" of the culture is a factor, as cells that were passaged many times can differ from recently thawed stocks $[59,60]$. RdRPs, which are crucial for small RNA inheritance in C.elegans, are not known to be conserved in humans [4], however, even if this mechanism is missing in other organisms, epigenetic memories could transmit to daughter cells via different feedback mechanisms $[61,62]$. For example, in human cells, genes known to be induced by IFN-y were found to exhibit a more robust induction in cells that have previously experienced IFN-Y [63,64]. Importantly, this effect was maintained for 4-7 cell divisions, and was associated with H3K4 dimethylation [64]. Therefore, while the causative agents are not always characterized, it is clear that some information which is not encoded in the DNA sequence can persist for long durations of time. This type of inheritance should be taken into account in the experimental design. 


\section{Methods}

\section{Cultivation of worms}

Standard culture techniques were used to maintain the nematodes on nematode growth medium (NGM) plates seeded with OP50 Escherichia coli bacteria. Extreme care was taken to avoid contamination or starvation, and contaminated plates were discarded from the analysis. For inheritance experiments worms were synchronized either by a standard Sodium hypochlorite bleaching techniques, or by letting adult worms lay eggs on OP50 seeded plates for two hours.

\section{Growth in liquid media}

Worm eggs were seeded into flasks with $20 \mathrm{~mL}$ of $\mathrm{M} 9$ buffer (including $1 \mathrm{mM}$ $\mathrm{MgSO}_{4},[18]$ ) supplemented with $200 \mu \mathrm{l}$ Penicillin-Streptomycin-Nystatin (Penicillin G Sodium Salt: 10,000 units $/ \mathrm{mL}$, Streptomycin Sulfate: $10 \mathrm{mg} / \mathrm{mL}$, Nystatin 1,250 units $/ \mathrm{mL}$ ), $20 \mu \mathrm{l}$ Cholesterol $(5 \mathrm{mg} / \mathrm{mL})$ and were fed with 200 microliter of $1 \mathrm{~mL}$ concentrated pellet of $50 \mathrm{~mL}$ OP51 E. coli bacteria that were previously grown over night (LB buffer + $100 \mu \mathrm{g} / \mathrm{mL}$ Streptomycin). The liquid flask was shaken (120 RPM) at $20^{\circ} \mathrm{C}$.

\section{L1 arrest experiments}

Adult worms were washed using M9 buffer [18]. After removal of the buffer supernatant, $\sim 100 \mu \mathrm{l}$ pellet of worms was treated for 5 minutes with $1 \mathrm{~mL}$ of bleach solution (2 $\mathrm{mL}$ of $5 \%$ sodium hypochlorite, $7.5 \mathrm{~mL}$ double distilled water, $0.5 \mathrm{~mL} \mathrm{NaOH}$ 10M). Next, $0.5 \mathrm{~mL}$ of M9 buffer was added and the solution was centrifuged (8000 RPM, 1 minutes). The resulting egg pellet was washed three times by removing the supernatant, 
adding fresh M9 buffer and centrifugation of 8000 RPM for 1 minutes to re-pellet the eggs. Around 400 eggs were left to hatch and arrest in the larval L1 stage on fresh plates for $\sim 12$ hours. Next, larval worms were transferred using M9 buffer to NGM plates seeded with OP50 bacteria. Worms were photographed 72 hours later.

\section{Strains used in this study:}

YY720: [hrde-1(tm1200) III.], YY158: [nrde-3(gg66) X.], RB798: [rrf-1(ok589) I.], GW638: [set-25(n5021) III. ; met-2(n4256) III.], VC967: [set-32(ok1457) I.], MT17463: [set-25(n5021) III.], WM27: [rde-1(ne219) V.], WM49: [rde-4(ne301) III.], YY13: [rrf3(mg373) II.], GR1823: [mut-16(mg461) I.], CF1038: [daf-16(mu86) I.]. CB1033: [che2(e1033) X.], DR476: [daf-22(m130) II.]. , EG6699: [ttTi5605 II. ; unc-119(ed3) III.], CX13249: [srg-37,srg-38 (kylR88) X.], CX13591: [srg-36,srg-38 (kylR95) X.]

\section{Worm length measurements}

Worms were placed on empty NGM plates and photographed using ScopeTek DCM310 binocular camera. The worms' length was measured using worm-sizer [65].

\section{Egg length measurements}

Worms were allowed to lay eggs on an empty NGM plate, the laid eggs were photographed using ScopeTek DCM310 binocular camera. Egg length was measured using the particle analysis tool of the ImageJ software. 


\section{Statistical Analysis}

Two-Way ANOVA was used to compare the effect two categorical variables on the length of the worm's progeny (this statistical analysis is relevant to the experiments shown on Figures 1D, 2A, 2C, and Figures S2B and S2C): A. biological condition (growth in liquid or on plates) and B. biological repeat (independent experiments conducted on separate groups of animals, on different days). One-Way ANOVA was used to compare the effect of growth in liquid medium on worm's length between different genetic backgrounds (Figures 3 and 4), between different food regimes (Figure 1C and Figure S1), or between conditions across generations (Figure 5). In cases of multiple comparisons between genotypes conditions, Sidak's multiple comparison test was applied. Linear regression analysis and a statistical method equivalent to analysis of covariance was used to compare the growth rates of progeny of liquid-grown worms and control worms throughout development (Figure 2B and Figure S3). Biological repeats are defined as independent experiments conducted on separate populations of animals, on different days. Statistical tests were performed using GraphPad Prism software (Graphpad Prism) version 6. 


\section{Data accessibility}

This article has no additional data.

\section{Competing interests}

We declare we have no competing interests.

\section{Author contributions}

I.L., R.B. and O.R. conceived and designed the experiments; I.L., R.B., Y.L. and L.C. performed the experiments; I.L., and O.R. wrote the paper.

\section{Acknowledgments}

We thank all the Rechavi lab members for the helpful comments and fruitful discussions. We thank Yoav Zeevi, Yoav Benjamini's group, for his help with the statistical analysis. Some strains were provided by the CGC, which is funded by NIH Office of Research Infrastructure Programs (P40 OD010440). We thank the ERC (grant \#335624), the Israel Science Foundation (grant \#1339/17) and the Allen Discovery Center program through The Paul G. Allen Frontiers Group (12171). O.R. gratefully acknowledges the support of the Adelis foundation (\#01430001000). 


\section{References:}

1. Radick G. 2015 Beyond the "Mendel-Fisher controversy". Science (80-. ). 350, 159 LP-160.

2. Anava S, Posner R, Rechavi O. 2014 The soft genome. Worm 3, e989798. (doi:10.4161/21624054.2014.989798)

3. Heard E, Martienssen RA. 2014 Transgenerational Epigenetic Inheritance: Myths and Mechanisms. Cell 157, 95-109. (doi:10.1016/j.cell.2014.02.045)

4. Rechavi O, Lev I. 2017 Principles of Transgenerational Small RNA Inheritance in Caenorhabditis elegans. Curr. Biol. 27, R720-R730.

(doi:10.1016/j.cub.2017.05.043)

5. Rechavi O, Minevich G, Hobert O. 2011 Transgenerational inheritance of an acquired small RNA-based antiviral response in C. elegans. Cell 147, 1248-56. (doi:10.1016/j.cell.2011.10.042)

6. Gammon DB, Ishidate T, Li L, Gu W, Silverman N, Mello CC. 2017 The Antiviral RNA Interference Response Provides Resistance to Lethal Arbovirus Infection and Vertical Transmission in Caenorhabditis elegans. Curr. Biol. 27, 795-806. (doi:10.1016/j.cub.2017.02.004)

7. Guo X, Li W-X, Lu R. 2012 Silencing of host genes directed by virus-derived short interfering RNAs in Caenorhabditis elegans. J. Virol. 86, 11645-53. (doi:10.1128/JVI.01501-12)

8. Rechavi O, Houri-Ze'evi L, Anava S, Goh WSS, Kerk SY, Hannon GJ, Hobert O. 2014 Starvation-Induced Transgenerational Inheritance of Small RNAs in C. elegans. Cell 158, 277-87. (doi:10.1016/j.cell.2014.06.020) 
9. Jobson MA, Jordan JM, Sandrof MA, Hibshman JD, Ashley L, Baugh LR. 2015 Transgenerational effects of early life starvation on growth , reproduction and stress resistance in C . elegans. Genetics 201, 1-37. (doi:10.1534/genetics.115.178699)

10. Demoinet E, Li S, Roy R. 2017 AMPK blocks starvation-inducible transgenerational defects in Caenorhabditis elegans. Proc. Natl. Acad. Sci. 114, E2689-E2698. (doi:10.1073/pnas.1616171114)

11. Webster AK, Jordan JM, Hibshman JD, Chitrakar R, Baugh LR. 2018 Transgenerational Effects of Extended Dauer Diapause on Starvation Survival and Gene Expression Plasticity in Caenorhabditis elegans. Genetics, genetics.301250.2018. (doi:10.1534/genetics.118.301250)

12. Klosin A, Casas E, Hidalgo-Carcedo C, Vavouri T, Lehner B. 2017 Transgenerational transmission of environmental information in C. elegans. Science (80-. ). 356, 320-323. (doi:10.1126/science.aah6412)

13. Schott D, Yanai I, Hunter CP. 2014 Natural RNA interference directs a heritable response to the environment. Sci. Rep. 4, 7387. (doi:10.1038/srep07387)

14. Ni JZ, Kalinava N, Chen E, Huang A, Trinh T, Gu SG. 2016 A transgenerational role of the germline nuclear RNAi pathway in repressing heat stress-induced transcriptional activation in C. elegans. Epigenetics Chromatin 9, 3. (doi:10.1186/s13072-016-0052-x)

15. Kishimoto S, Uno M, Okabe E, Nono M, Nishida E. 2017 Environmental stresses induce transgenerationally inheritable survival advantages via germline-to-soma communication in Caenorhabditis elegans. Nat. Commun. 8, 14031. 
(doi:10.1038/ncomms14031)

16. Burton NO, Furuta T, Webster AK, Kaplan REW, Baugh LR, Arur S, Horvitz HR. 2017 Insulin-like signalling to the maternal germline controls progeny response to osmotic stress. Nat. Cell Biol. (doi:10.1038/ncb3470)

17. Tauffenberger A, Parker JA. 2014 Heritable Transmission of Stress Resistance by High Dietary Glucose in Caenorhabditis elegans. PLoS Genet. 10, e1004346.

(doi:10.1371/journal.pgen.1004346)

18. Stiernagle T. 2006 Maintenance of C. elegans. The C. elegans Research Community. (doi:10.1895/wormbook.1.101.1)

19. Szewczyk NJ, Kozak E, Conley CA. 2003 Chemically defined medium and Caenorhabditis elegans. BMC Biotechnol. 3, 19. (doi:10.1186/1472-6750-3-19)

20. Laranjeiro R, Harinath G, Burke D, Braeckman BP, Driscoll M. 2017 Single swim sessions in C. elegans induce key features of mammalian exercise. BMC Biol. 15, 30. (doi:10.1186/s12915-017-0368-4)

21. Han R, Ehlers R-U. 2000 Pathogenicity, Development, and Reproduction of Heterorhabditis bacteriophora and Steinernema carpocapsae under Axenic in Vivo Conditions. J. Invertebr. Pathol. 75, 55-58.

22. Peters A, Han R, Yan X, Leite LG. 2017 Production of Entomopathogenic Nematodes. Microb. Control Insect Mite Pests , 157-170. (doi:10.1016/B978-0$12-803527-6.00010-X)$

23. Surrey MR, Davies RJ. 1996 Pilot-scale liquid culture and harvesting of an entomopathogenic nematode, Heterorhabditis bacteriophora. J. Invertebr. Pathol. 67, 92-99. (doi:10.1006/jipa.1996.0013) 
24. Upadhyay D, Mandjiny S, Bullard-Dillard R, Storms M, Menefee M, Holmes LD.

2015 Lab-scale in vitro Mass Production of the Entomopathogenic Nematode

Heterorhabditis bacteriophora Using Liquid Culture Fermentation Technology.

Am. J. Biosci. Bioeng. 3, 203. (doi:10.11648/j.bio.20150306.19)

25. Werner MS, Sieriebriennikov B, Loschko T, Namdeo S, Lenuzzi M, Dardiry M,

Renahan T, Sharma DR, Sommer RJ. 2017 Environmental influence on

Pristionchus pacificus mouth form through different culture methods. Sci. Rep. 7,

7207. (doi:10.1038/s41598-017-07455-7)

26. Kiontke K. 2006 Ecology of Caenorhabditis species. WormBook

(doi:10.1895/wormbook.1.37.1)

27. Schulenburg H, Félix M-A. 2017 The Natural Biotic Environment of

Caenorhabditis elegans. Genetics 206, 55-86. (doi:10.1534/genetics.116.195511)

28. Frøkjær-Jensen C et al. 2016 An Abundant Class of Non-coding DNA Can

Prevent Stochastic Gene Silencing in the C. elegans Germline. Cell 166, 343-

357. (doi:10.1016/j.cell.2016.05.072)

29. Timmons L, Tabara H, Mello CC, Fire AZ. 2003 Inducible systemic RNA silencing in Caenorhabditis elegans. Mol. Biol. Cell 14, 2972-83. (doi:10.1091/mbc.E03-010858)

30. Doh JH, Moore AB, Celen I, Moore MT, Sabanayagam CR. 2016 ChIP and Chips: Introducing the WormPharm for correlative studies employing pharmacology and genome-wide analyses in C. elegans. J. Biol. Methods 3, 44.

(doi:10.14440/jbm.2016.109)

31. Çelen İ, Doh JH, Sabanayagam CR. 2018 Effects of liquid cultivation on gene 
expression and phenotype of C. elegans. BMC Genomics 19, 562.

(doi:10.1186/s12864-018-4948-7)

32. Kaplan F et al. 2011 Ascaroside Expression in Caenorhabditis elegans Is Strongly Dependent on Diet and Developmental Stage. PLoS One 6, e17804.

(doi:10.1371/journal.pone.0017804)

33. von Reuss SH, Bose N, Srinivasan J, Yim JJ, Judkins JC, Sternberg PW, Schroeder FC. 2012 Comparative Metabolomics Reveals Biogenesis of Ascarosides, a Modular Library of Small-Molecule Signals in C. elegans. J. Am. Chem. Soc. 134, 1817-1824. (doi:10.1021/ja210202y)

34. Ludewig $\mathrm{AH}$ et al. 2017 Larval crowding accelerates C. elegans development and reduces lifespan. PLOS Genet. 13, e1006717.

(doi:10.1371/journal.pgen.1006717)

35. Hu PJ. 2007 Dauer. WormBook (doi:10.1895/wormbook.1.144.1)

36. Srinivasan J et al. 2008 A blend of small molecules regulates both mating and development in Caenorhabditis elegans. Nature 454, 1115-1118.

(doi:10.1038/nature07168)

37. Golden JW, Riddle DL. 1985 A gene affecting production of the Caenorhabditis elegans dauer-inducing pheromone. MGG Mol. Gen. Genet. 198, 534-536. (doi:10.1097/01.EPX.0000417960.79703.06)

38. McGrath PT, Xu Y, Ailion M, Garrison JL, Butcher RA, Bargmann CI. 2011 Parallel evolution of domesticated Caenorhabditis species targets pheromone receptor genes. Nature 477, 321-325. (doi:10.1038/nature10378)

39. Lewis JA, Hodgkin JA. 1977 Specific neuroanatomical changes in chemosensory 
mutants of the nematodeCaenorhabditis elegans. J. Comp. Neurol. 172, 489-510. (doi:10.1002/cne.901720306)

40. Perkins LA, Hedgecock EM, Thomson JN, Culotti JG. 1986 Mutant sensory cilia in the nematode Caenorhabditis elegans. Dev. Biol. 117, 456-87.

41. Bargmann Cl, Hartwieg E, Robert Horvitz H. 1993 Odorant-Selective Genes and Neurons Mediate Olfaction in C. elegans. Cell. 74.

42. Murphy CT, Hu PJ. 2013 Insulin/insulin-like growth factor signaling in C. elegans. WormBook , 1-43. (doi:10.1895/wormbook.1.164.1)

43. Buckley B a, Burkhart KB, Gu SG, Spracklin G, Kershner A, Fritz H, Kimble J, Fire A, Kennedy S. 2012 A nuclear Argonaute promotes multigenerational epigenetic inheritance and germline immortality. Nature 489, 447-51.

(doi:10.1038/nature11352)

44. Guang S, Bochner AF, Pavelec DM, Burkhart KB, Harding S, Lachowiec J, Kennedy S. 2008 An Argonaute transports siRNAs from the cytoplasm to the nucleus. Science 321, 537-41. (doi:10.1126/science.1157647)

45. Burton NO, Burkhart KB, Kennedy S. 2011 Nuclear RNAi maintains heritable gene silencing in Caenorhabditis elegans. Proc. Natl. Acad. Sci. 108, 19683-8. (doi:10.1073/pnas.1113310108)

46. Sapetschnig A, Sarkies P, Lehrbach NJ, Miska EA. 2015 Tertiary siRNAs Mediate Paramutation in C. elegans. PLoS Genet. 11. (doi:10.1371/journal.pgen.1005078)

47. Gu SG, Pak J, Guang S, Maniar JM, Kennedy S, Fire A. 2012 Amplification of siRNA in Caenorhabditis elegans generates a transgenerational sequencetargeted histone H3 lysine 9 methylation footprint. Nat. Genet. 44, 157-64. 
(doi:10.1038/ng.1039)

48. Lev I, Gingold H, Rechavi O. $2018 \mathrm{H} 3 \mathrm{~K} 9 \mathrm{me} 3$ is Required for Transgenerational Inheritance of Small RNAs that Target a Unique Subset of Newly Evolved Genes.

Elife accepted f, 338582. (doi:10.1101/338582)

49. Lev I, Seroussi U, Gingold H, Bril R, Anava S, Rechavi O. 2017 MET-2Dependent H3K9 Methylation Suppresses Transgenerational Small RNA Inheritance. Curr. Biol. 27, 1138-1147. (doi:10.1016/j.cub.2017.03.008)

50. McMurchy AN et al. 2017 A team of heterochromatin factors collaborates with small RNA pathways to combat repetitive elements and germline stress. Elife 6, e21666. (doi:10.7554/eLife.21666)

51. Towbin BDD, González-Aguilera C, Sack R, Gaidatzis D, Kalck V, Meister P, Askjaer P, Gasser SMM. 2012 Step-wise methylation of histone H3K9 positions heterochromatin at the nuclear periphery. Cell 150, 934-947.

(doi:10.1016/j.cell.2012.06.051)

52. Spracklin G, Fields B, Wan G, Vijayendran D, Wallig A, Shukla A, Kennedy S. 2017 Identification and Characterization of C. elegans RNAi Inheritance Machinery. Genetics , 1-19. (doi:10.20944/preprints201702.0096.v1)

53. Rechavi O, Houri-Ze'evi L, Anava S, Goh WSS, Kerk SY, Hannon GJ, Hobert O. 2014 Starvation-Induced Transgenerational Inheritance of Small RNAs in C. elegans. Cell 158, 277-287. (doi:10.1016/j.cell.2014.06.020)

54. Hodgkin J, Félix M-A, Clark LC, Stroud D, Gravato-Nobre MJ. 2013 Two Leucobacter Strains Exert Complementary Virulence on Caenorhabditis Including Death by Worm-Star Formation. Curr. Biol. 23, 2157-2161. 
(doi:10.1016/j.cub.2013.08.060)

55. Ehlers R-U, Lunau S, Krasomil-Osterfeld K, Osterfeld KH. 1998 Liquid culture of the entomopathogenic nematode-bacterium-complex Heterorhabditis megidis/Photorhabdus luminescens. BioControl 43, 77-86.

(doi:10.1023/A:1009965922794)

56. Strauch O, Ehlers R-U. 1998 Food signal production of Photorhabdus luminescens inducing the recovery of entomopathogenic nematodes Heterorhabditis spp. in liquid culture. Appl. Microbiol. Biotechnol. 50, 369-374. (doi:10.1007/s002530051306)

57. Capes-Davis A et al. 2010 Check your cultures! A list of cross-contaminated or misidentified cell lines. Int. J. Cancer 127, 1-8. (doi:10.1002/ijc.25242)

58. Ben-David U et al. 2018 Genetic and transcriptional evolution alters cancer cell line drug response. Nature 560, 325-330. (doi:10.1038/s41586-018-0409-3)

59. Merrill GF. 1998 Cell synchronization. Methods Cell Biol. 57, 229-49.

60. Niida H, Matsumoto T, Satoh H, Shiwa M, Tokutake Y, Furuichi Y, Shinkai Y. 1998 Severe growth defect in mouse cells lacking the telomerase RNA component. Nat. Genet. 19, 203-6. (doi:10.1038/580)

61. Campos El, Stafford JM, Reinberg D. 2014 Epigenetic inheritance: histone bookmarks across generations. Trends Cell Biol. 24, 664-74. (doi:10.1016/j.tcb.2014.08.004)

62. Wang $X$ et al. 2013 Tissue culture-induced genetic and epigenetic alterations in rice pure-lines, F1 hybrids and polyploids. BMC Plant Biol. 13, 77. (doi:10.1186/1471-2229-13-77) 
63. Gialitakis M, Arampatzi P, Makatounakis T, Papamatheakis J. 2010 Gamma interferon-dependent transcriptional memory via relocalization of a gene locus to PML nuclear bodies. Mol. Cell. Biol. 30, 2046-56. (doi:10.1128/MCB.00906-09)

64. Light WH, Freaney J, Sood V, Thompson A, D'Urso A, Horvath CM, Brickner JH. 2013 A Conserved Role for Human Nup98 in Altering Chromatin Structure and Promoting Epigenetic Transcriptional Memory. PLoS Biol. 11, e1001524. (doi:10.1371/journal.pbio.1001524)

65. Moore BT, Jordan JM, Baugh LR. 2013 WormSizer: high-throughput analysis of nematode size and shape. PLoS One 8. (doi:10.1371/journal.pone.0057142) 


\section{Supplementary Figures:}

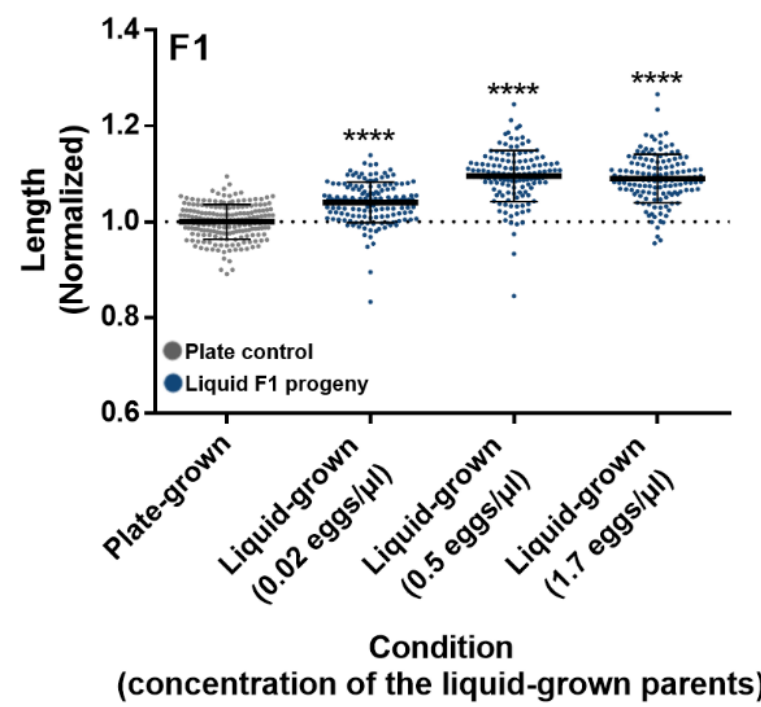

Supplementary Figure 1. F1 progeny of liquid grown worms are longer irrespectively of the concentration in which their liquid-grown parents were grown.

The normalized lengths of $\mathrm{F} 1$ progeny of worms grown in liquid in different concentrations (x-axis) and progeny of plate-grown controls are presented. The measured length is normalized to the corresponding control progeny of plate-grown worms. ${ }^{* * *} p$-value $<0.0001$. One-way ANOVA, Sidak's multiple comparisons test. Error bars represent standard deviations. 
A

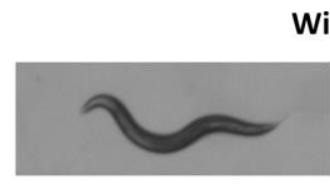

Plate grown (P0)
Wild type

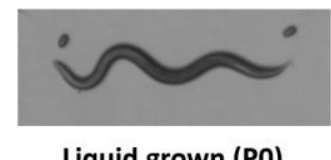

unc-119 mutants

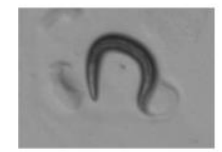

Plate grown (PO)

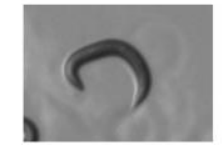

Liquid grown (P0)
B

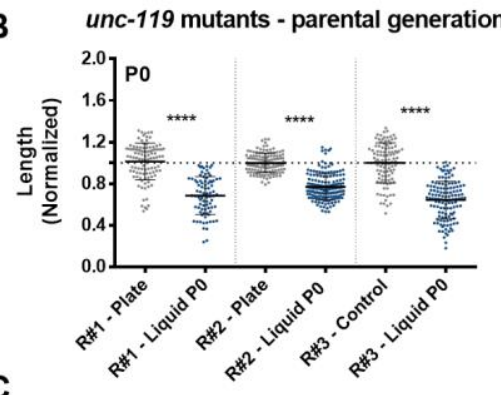

C

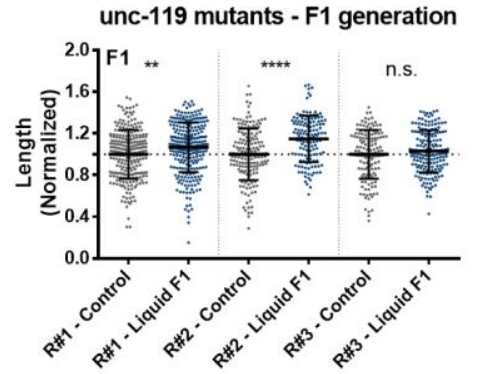

\section{Supplementary Figure 2. Immobile mutant worms do not alter their morphology} when grown in liquid, but their progeny are longer.

(A) Representative photographs of plate- and liquid- grown wild type and unc-119 adult worms. (B) Quantification of the length of the parental unc-119 mutants grown either on plates or in liquid. The measured length was normalized to the corresponding control worms. Data from three independent biological repeats are presented $(\mathrm{N}>80) .{ }^{* * *} \mathrm{p}-$ value $<0.0001$, Sidak's multiple comparisons test. (C) Plate-grown progeny of immobile unc-119 mutant worms are longer than plate grown controls. The length of F1 plate-grown progeny of liquid-grown worms and progeny of plate-grown controls are shown. The measured length was normalized to the corresponding control worms. Data from three independent biological repeats are presented ( $N>130$ worms per group). The $p$-value was determined using Two-way ANOVA with the two categorical variables: A. condition (growth in liquid or on plates) and B. biological repeat. The biological condition factor is 
significant ( $p$-value $<0.0001)$. The biological repeat factor and interaction effect are also significant ( $p=0.0024$ for both). The biological condition factor explains more variance $(2.76 \%)$ than the biological repeat or the interaction effect factors $(1 \%)$. ${ }^{* * *} p-$ value $<0.0001,{ }^{* *} p$-value $=0.0027$, Sidak's multiple comparisons test.

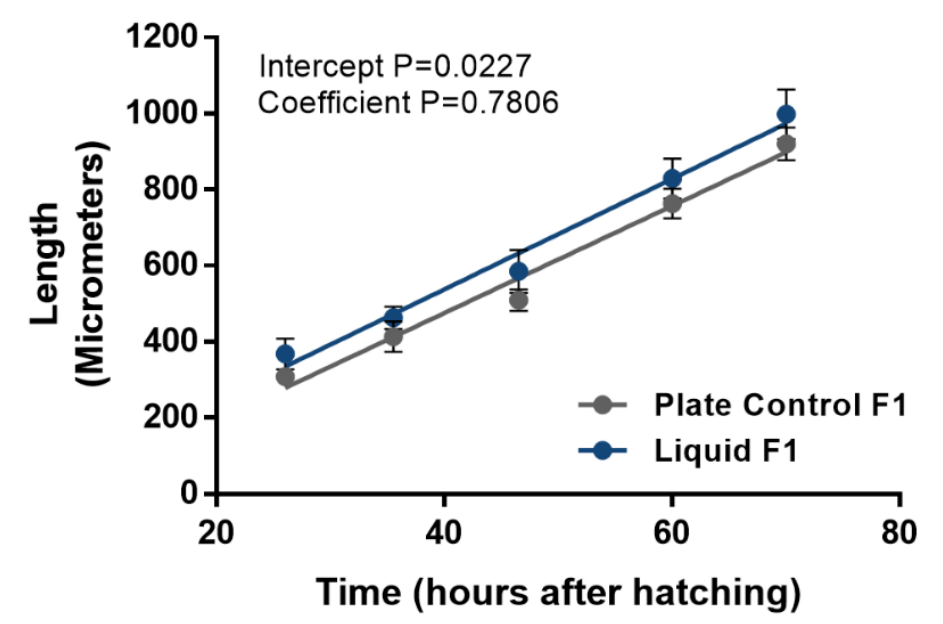

Supplementary Figure 3. Linear regression analysis of growth rates of progeny of liquid-grown and control worms.

Linear regression analysis of the length of progeny of liquid-grown worms and control worms at different time-points during the worm's development. While the intercepts are significantly different $(p=0.022)$, the rate of growth, or regression coefficients are identical $(p=0.7806)$. Error bars represent standard deviations. 